\title{
ULRICH BUNDLES ON RULED SURFACES
}

\author{
MARIAN APRODU, LAURA COSTA, ROSA MARIA MIRÓ-ROIG
}

\begin{abstract}
In this short note, we study the existence problem for Ulrich bundles on ruled surfaces, focusing our attention on the smallest possible rank. We show that existence of Ulrich line bundles occurs if and only if the coefficient $\alpha$ of the minimal section in the numerical class of the polarization equals one. For other polarizations, we prove the existence of rank two Ulrich bundles.
\end{abstract}

\section{INTRODUCTION}

Let $(X, H)$ be a smooth complex projective variety of dimension $n$. An Ulrich bundle on $X$ is a vector bundle $E$ which satisfies $H^{i}(X, E(-i H))=0$ for all $i>0$ and $H^{j}\left(X, E(-(j+1) H)=0\right.$ for all $j<n$. Its pushforward to $\mathbb{P}^{m}$ via the inclusion of $X$ given by the ample line bundle $H$ has a linear resolution or, equivalently, for any linear projection $X \rightarrow \mathbb{P}^{n}$, its pushforward is trivial (see ES03, Proposition 2.1).

Ulrich bundles have made a first appearance in commutative algebra, being associated to maximal Cohen-Macaulay graded modules with maximal number of generators, [U184]. In algebraic geometry, their importance was underlined in Bea00] and ES03 where a relation between their existence and the representations of Cayley-Chow forms was found. They have also important applications in liaison theory, singularity theory, moduli spaces and Boij-Söderberg theory. For example, if $X$ admits an Ulrich bundle, then its cone of cohomology tables coincide with that of $\mathbb{P}^{n}$ [ES11]. In view of their importance, Eisenbud, Schreyer and Weyman formulated the problem of determining which varieties $X$ admits an Ulrich bundle and, if so, what is the smallest rank of an Ulrich bundle that they carry. So far few examples are known and we refer the reader to [CH12, CKM12, [CKM13, [CMR], CMP], ES03, [KM], [Mi], [MP and [MP2] for references and further results. In this context the existence of undecomposable rank 2 Ulrich bundles on a surfaces is a challenging problem and a particular attention is focused on the existence of special rank two Ulrich bundles, i.e. rank 20 -regular vector bundles $E$ on $X$ such that $c_{1}(E)=K_{X}+3 H$. The existence of a special Ulrich bundle implies that the associated Cayley-Chow form is represented as a linear pfaffian ES03. Recently, this problem has been solved for general K3 surfaces [AFO12, [CKM12, for del Pezzo surfaces in MP2, for ACM rational surfaces in MP] and for surfaces with $q=p_{g}=0$, Bea16]. We will focus here on surfaces with Kodaira dimension $-\infty$. In this note, we study the existence problem for Ulrich bundles on ruled surfaces, which is a relevant case thanks to [Kim16].

Date: February 14, 2018.

Marian Aprodu thanks the Max Planck Institute for Mathematics Bonn for hospitality during the preparation of this work The second and third authors were partially supported by MTM2013-45075-P. 
The structure of the paper is the following. In Section 2 we analyze the existence of Ulrich line bundles. We show that existence occurs if and only if the coefficient $\alpha$ of the minimal section in the numerical class of the polarization equals one, Theorem 2.1. In Section 3 we prove existence of rank two special Ulrich bundles with respect to arbitrary polarizations (Proposition 3.1, Proposition 3.3 and Theorem 3.4). The rank-two bundles that we construct are strictly semistable for $\alpha=1$ and stable for $\alpha \geq 2$, Remark 3.7 For basic properties of Ulrich bundles, we refer to the literature included here, especially ES03.

Notation. $\pi: X \rightarrow C$ is a geometrically ruled surface of invariant $e>0$ over the genus$g$ curve $C$. Fix $p \in C$ and denote $F$ the fibre of $\pi$ over $p$. Denote by $C_{0}$ the section of self-intersection $-e$. Write $\mathcal{O}_{X}\left(K_{X}\right)=\mathcal{O}_{X}\left(-2 C_{0}+(2 g-2-e) F\right) \otimes \pi^{*} \mathfrak{k}$, with $\mathfrak{k} \in \operatorname{Pic}^{0}(C)$. We shall work with a polarization $H_{\alpha}$ on $X$ of numerical class $H \equiv \alpha C_{0}+\beta F$ with $\alpha \geq 1$ and $\beta>\alpha e$.

\section{ULRICH LINE BUNDLES}

In this section, we analyze the existence of Ulrich line bundles. We note first that, if they exists, Ulrich line bundles come in pairs. Indeed, if $\mathcal{O}_{X}(L)$ is an Ulrich line bundle on $X$ with respect to the very ample line bundle $\mathcal{O}_{X}\left(H_{\alpha}\right)$ then

$$
\mathcal{O}_{X}\left(K_{X}+3 H_{\alpha}-L\right)
$$

is also an Ulrich line bundle on $X$. In fact, $\mathcal{O}_{X}\left(K_{X}+3 H_{\alpha}-L\right)$ is an Ulrich line bundle if and only if for any $i \geq 0$ and $-2 \leq t \leq-1, H^{i}\left(\mathcal{O}_{X}\left(K_{X}+3 H_{\alpha}-L-t H_{\alpha}\right)\right)=0$. Hence the claim follows from the fact that since $\mathcal{O}_{X}(L)$ is an Ulrich line bundle, for any $i \geq 0$ and $-2 \leq t \leq-1, H^{i}\left(\mathcal{O}_{X}\left(L-t H_{\alpha}\right)\right)=0$ and from the fact that from Serre's duality we have

$$
H^{i}\left(\mathcal{O}_{X}\left(K_{X}+3 H_{\alpha}-L-t H_{\alpha}\right)\right)=H^{2-i}\left(\mathcal{O}_{X}\left(L+(t-3) H_{\alpha}\right)\right)=0 .
$$

The main result of this section is the following.

Theorem 2.1. Let $X$ be a geometrically ruled surface over a smooth curve $C$ of genus $g$ and with $e>0$. Let $H_{\alpha}=\alpha C_{0}+\beta F$ be any very ample divisor on $X$. Then, there are Ulrich line bundles with respect to $H_{\alpha}$ if and only if $\alpha=1$. In this case, there exists exactly two types of Ulrich line bundles on $X$, namely

$$
\mathcal{O}_{X}\left(L_{1}\right):=\mathcal{O}_{X}((2 \beta+g-1-e) F) \otimes \pi^{*} \mathcal{L}_{1}
$$

and

$$
\mathcal{O}_{X}\left(L_{2}\right):=\mathcal{O}_{X}\left(K_{X}+3 H_{\alpha}-L_{1}\right)=\mathcal{O}_{X}\left(C_{0}+(\beta+g-1) F\right) \otimes \pi^{*} \mathcal{L}_{2}
$$

with $\mathcal{L}_{1}, \mathcal{L}_{2} \in \operatorname{Pic}^{0}(C)$ general.

Proof. Let $\mathcal{O}_{X}\left(L_{1}\right)$ be an Ulrich line bundle on $X$. Then its pair $\mathcal{O}_{X}\left(L_{2}\right):=\mathcal{O}_{X}\left(K_{X}+\right.$ $\left.3 H_{\alpha}-L_{1}\right)$ is also Ulrich. In particular, this means that there exists two divisors $D_{i}=$ $L_{i}-L \equiv a_{i} C_{0}+b_{i} F$, i.e. $\mathcal{O}_{X}\left(D_{i}\right)=\mathcal{O}_{X}\left(a_{i} C_{0}+b_{i} F\right) \otimes \pi^{*} \mathcal{L}_{i}$ for $a_{i}, b_{i} \in \mathbb{Z}$ and $\mathcal{L}_{i} \in \operatorname{Pic}^{0}(C)$, such that $\chi\left(\mathcal{O}_{X}\left(D_{i}\right)\right)=0, i \in\{1,2\}$. By Riemann-Roch's theorem this means that

$$
0=\chi\left(\mathcal{O}_{X}\left(D_{i}\right)\right)=1-g-\frac{D_{i} \cdot K_{X}}{2}+\frac{D_{i}^{2}}{2}=\left(a_{i}+1\right)\left(b_{i}+1-g-\frac{a_{i} e}{2}\right) .
$$

Therefore $a_{i}=-1$ or $b_{i}=g-1+\frac{a_{i} e}{2}$ for $i \in\{1,2\}$. Note that $a_{1}+a_{2}=\alpha-2$ and $b_{1}+b_{2}=\beta+2 g-2-e$. 
We claim that the equations $b_{i}=g-1+\frac{a_{i} e}{2}$ cannot be satisfied simultaneously. Indeed, if the two conditions are satisfied in the same time, then, by adding them up and replacing $b_{1}+b_{2}$ and $a_{1}+a_{2}$ we obtain $\beta=\alpha e$ which contradicts the very ampleness of $H_{\alpha}$.

On the other hand, since $a_{1}+a_{2}=\alpha-2, a_{1}$ and $a_{2}$ cannot be simultaneously -1 , for that would imply $\alpha=0$, contradiction.

Hence either $a_{1}=-1$ and $b_{2}=g-1+\frac{a_{2} e}{2}$ or $a_{2}=-1$ and $b_{1}=g-1+\frac{a_{2} e}{2}$.

Assume that $a_{1}=-1$ and $b_{2}=g-1+\frac{a_{2} e}{2}$ (the second case is similar, due to the symmetry of the situation). Then $a_{2}=\alpha-1$ and $b_{2}=g-1+\frac{(\alpha-1) e}{2}$, hence

$$
\mathcal{O}_{X}\left(L_{2}-H_{\alpha}\right)=\mathcal{O}_{X}\left((\alpha-1) C_{0}+\left(g-1+\frac{e(\alpha-1)}{2}\right) F\right) \otimes \pi^{*} \mathcal{L}_{2}
$$

Since $e \geq 1, \alpha \geq 1$, and $h^{0}\left(\mathcal{O}_{X}\left(L_{2}-H_{\alpha}\right)\right)=0$ it follows that $\alpha=1$. Indeed, if $\alpha \geq 2$, the degree of $\mathcal{O}_{C}\left(\left(g-1+\frac{e(\alpha-1)}{2}\right) p\right) \otimes \mathcal{L}_{2}$ is at least $g$ which means that $\mathcal{O}_{X}\left(\left(g-1+\frac{e(\alpha-1)}{2}\right) F\right) \otimes \pi^{*} \mathcal{L}_{2}$ has sections, implying also that the line bundle

$$
\mathcal{O}_{X}\left((\alpha-1) C_{0}+\left(g-1+\frac{e(\alpha-1)}{2}\right) F\right) \otimes \pi^{*} \mathcal{L}_{2}
$$

has sections. In conclusion, $\alpha=1$, which implies $\mathcal{O}_{X}\left(L_{1}\right)=\mathcal{O}_{X}((2 \beta+g-1-e) F) \otimes \pi^{*} \mathcal{L}_{1}$.

Conversely, assume that $\alpha=1$ and we prove that

$$
\mathcal{O}_{X}\left(L_{1}\right):=\mathcal{O}_{X}((2 \beta+g-1-e) F) \otimes \pi^{*} \mathcal{L}_{1},
$$

with $\mathcal{L}_{1} \in \operatorname{Pic}^{0}(C)$ general, is an Ulrich bundle. To this end, observe that

$$
H_{\alpha} \cdot L_{1}=\frac{H_{\alpha} \cdot\left(K_{X}+3 H_{\alpha}\right)}{2}
$$

and that by Riemann-Roch's Theorem

$$
\chi\left(\mathcal{O}_{X}\left(L_{1}-H_{\alpha}\right)\right)=(1-g)-\frac{1}{2}\left(L_{1}-H_{\alpha}\right) \cdot K_{X}+\frac{1}{2}\left(L_{1}-H_{\alpha}\right)^{2}=0 .
$$

These two facts also imply that $\chi\left(\mathcal{O}_{X}\left(L_{1}-2 H_{\alpha}\right)\right)=0$ because

$$
\chi\left(\mathcal{O}_{X}\left(L_{1}-2 H_{\alpha}\right)\right)=\chi\left(\mathcal{O}_{X}\left(L_{1}-H_{\alpha}\right)\right)-H_{\alpha} \cdot L_{1}+\frac{H_{\alpha} \cdot\left(K_{X}+3 H_{\alpha}\right)}{2} .
$$

On the other hand, $\mathcal{O}_{X}\left(L_{1}\right)$ is an Ulrich line bundle if and only if for any $i \geq 0$ and any $-2 \leq t \leq-1$, we have $H^{i}\left(\mathcal{O}_{X}\left(L_{1}-t H_{\alpha}\right)\right)=0$. Notice that the coefficient of $C_{0}$ in $L_{1}-H_{\alpha}$ equals -1 , hence $h^{0}\left(\mathcal{O}_{X}\left(L_{1}-H_{\alpha}\right)\right)=0$ which also implies that $h^{0}\left(\mathcal{O}_{X}\left(L_{1}-2 H_{\alpha}\right)\right)=$ 0 . The coefficient of $C_{0}$ in $H_{\alpha}-L_{1}+K_{X}$ equals also -1 and hence $h^{2}\left(\mathcal{O}_{X}\left(L_{1}-\right.\right.$ $\left.\left.H_{\alpha}\right)\right)=h^{0}\left(\mathcal{O}_{X}\left(H_{\alpha}-L_{1}+K_{X}\right)\right)=0$. These vanishing together with the fact that $\chi\left(\mathcal{O}_{X}\left(L_{1}-H_{\alpha}\right)\right)=0$ give us $h^{1}\left(\mathcal{O}_{X}\left(L_{1}-H_{\alpha}\right)\right)=0$. Finally, $\mathcal{O}_{X}\left(2 H_{\alpha}-L_{1}+K_{X}\right)=$ $\mathcal{O}_{X}((g-1) F) \otimes \pi^{*} \mathcal{L}_{2}$ with $\mathcal{L}_{2} \in \operatorname{Pic}^{0}(C)$ general and hence

$$
h^{2}\left(\mathcal{O}_{X}\left(L_{1}-2 H_{\alpha}\right)\right)=h^{0}\left(\mathcal{O}_{X}\left(2 H_{\alpha}-L_{1}+K_{X}\right)\right)=h^{0}\left(\mathcal{O}_{C}((g-1) p) \otimes \mathcal{L}_{2}\right)=0 .
$$

Since $\chi\left(\mathcal{O}_{X}\left(L_{1}-2 H_{\alpha}\right)\right)=0$ we also have $h^{1}\left(\mathcal{O}_{X}\left(L_{1}-2 H_{\alpha}\right)\right)=0$. Putting altogether we get that $\mathcal{O}_{X}\left(L_{1}\right)$ is an Ulrich line bundle and hence the same holds for $\mathcal{O}_{X}\left(L_{2}\right)$. 


\section{RANK-TWO UlRICH BUNDLES}

The goal of this section is to prove the existence of special rank 2 Ulrich bundles with respect to any very ample divisor $H_{\alpha}=\alpha C_{0}+\beta F$ on $X$. To this end, we will start analyzing separately the cases $\alpha=1$ and $\alpha=2$.

Proposition 3.1. Let $X$ be a geometrically ruled surface over a smooth curve $C$ of genus $g$ and with $e>0$. Let $H=C_{0}+\beta F$ be a very ample divisor on $X$. Then there exists a family of dimension $2 \beta-e+4 g-3$ of indecomposable rank-two simple strictly semi-stable Ulrich bundles on $X$.

Proof. It follows from Theorem 2.1 that

$$
\mathcal{O}_{X}\left(L_{1}\right):=\mathcal{O}_{X}((2 \beta-1-e) F) \otimes \pi^{*} \mathcal{L}_{1} \quad \text { and } \quad \mathcal{O}_{X}\left(L_{2}\right):=\mathcal{O}_{X}\left(K_{X}+3 H-L_{1}\right) \otimes \pi^{*} \mathcal{L}_{2}
$$

are Ulrich line bundles on $X$ for general $\mathcal{L}_{1} \in P i c^{0}(C)$ and $\mathcal{L}_{2}=\mathfrak{k} \otimes \mathcal{L}_{1}^{-1}$. Moreover, $h^{0}\left(\mathcal{O}_{X}\left(L_{2}-L_{1}\right)\right)=h^{2}\left(\mathcal{O}_{X}\left(L_{2}-L_{1}\right)\right)=0$ and hence

$\operatorname{dim} \operatorname{Ext}^{1}\left(\mathcal{O}_{X}\left(L_{1}\right), \mathcal{O}_{X}\left(L_{2}\right)\right)=h^{1}\left(\mathcal{O}_{X}\left(L_{2}-L_{1}\right)\right)=-\chi\left(\mathcal{O}_{X}\left(L_{2}-L_{1}\right)\right)=2 \beta-e+2(g-1)>0$.

Therefore we can consider rank two vector bundles $E$ on $X$ given by a non trivial extension of the following type

$$
0 \rightarrow \mathcal{O}_{X}\left(C_{0}+(\beta-1) F\right) \otimes \pi^{*} \mathcal{L}_{2} \rightarrow E \rightarrow \mathcal{O}_{X}((2 \beta-1-e) F) \otimes \pi^{*} \mathcal{L}_{1} \rightarrow 0
$$

Since $\mathcal{O}_{X}\left(L_{1}\right) \otimes \pi^{*} \mathcal{L}_{1}$ and $\mathcal{O}_{X}\left(L_{2}\right) \otimes \pi^{*} \mathcal{L}_{2}$ are Ulrich line bundles, $E$ is also an Ulrich bundle. Moreover it is simple and in particular indecomposable due to the fact that there are no nonzero morphisms from $L_{i}$ to $L_{j}$ for $i \neq j$. Finally notice that since Ulrich bundles are semi-stable and the slope of $L_{2}$ and $E$ coincide, it is strictly semi-stable.

Finally, since $h^{0}\left(E\left(-C_{0}-(\beta-1) F\right)\right)=1$, we have a family of dimension $2 \beta-e+4 g-3$ of indecomposable rank-two simple strictly semi-stable Ulrich bundles on $X$.

Before we pass to the case where the coefficient of $C_{0}$ in the polarization $H_{\alpha}$ is equal or greater than two, we will prove the following result that can be stated in a more general setting:

Lemma 3.2. Let $S$ be a surface, $H$ be a very ample divisor on $S, E$ a rank-two vector bundle on $S$ with $\operatorname{det}(E)=\mathcal{O}_{S}\left(K_{S}+3 H\right)$ and $c_{2}(E)=\frac{1}{2} H \cdot\left(5 H+3 K_{S}\right)+2 \chi\left(\mathcal{O}_{S}\right)$. Then $E$ is Ulrich if and only if $E$ is initialized i.e. $H^{0}(E(-H))=0$.

Proof. If $E$ is Ulrich, then by definition $H^{0}(E(-H))=0$ and hence we only have to prove the converse. For surfaces, Ulrichness of $E$ reduces to

$$
h^{0}(E(-H))=h^{1}(E(-H))=h^{1}(E(-2 H))=h^{2}(E(-2 H))=0 .
$$

On the other hand, $E^{*} \cong E\left(-K_{S}-3 H\right)$ and hence Serre's duality gives $h^{2}(E(-2 H))=$ $h^{0}\left(E^{*}\left(K_{S}+2 H\right)\right)=h^{0}(E(-H))$. Note also that the vanishing of $h^{0}(E(-H))$ implies the vanishing of $h^{0}(E(-2 H))\left(H^{0}(E(-2 H)) \subset H^{0}(E(-H))\right)$ and of $h^{2}(E(-H))$ by Serre duality.

The vanishing of $h^{1}(E(-H))$ and of $h^{1}(E(-2 H))$ follows if we prove the vanishing of $\chi(E(-H))$ and of $\chi(E(-2 H))$ respectively. 
Notice that $\operatorname{det}(E(-H))=K_{S}+H$ and $\operatorname{det}(E(-2 H))=K_{S}-H$. On the other hand, $c_{2}(E(-H))=c_{2}(E)-c_{1}(E) \cdot H+H^{2}$ and $c_{2}(E(-2 H))=c_{2}(E)-2 c_{1}(E) \cdot L+4 H^{2}$. Thus, from Riemann-Roch

$$
\chi(E(-H))=2 \chi\left(\mathcal{O}_{S}\right)+\frac{1}{2} c_{1}(E(-H)) \cdot\left(c_{1}(E(-H))-K_{S}\right)-c_{2}(E(-H))=0
$$

and similarly $\chi(E(-2 H))=0$.

Let us now consider the case where the coefficient of $C_{0}$ in the polarization $H_{\alpha}$ is equal to two:

Proposition 3.3. Let $X$ be a geometrically ruled surface over a smooth curve $C$ of genus $g$ and with $e>0$. Let $H=2 C_{0}+\beta F$ be any very ample divisor on $X$. Then, there are special Ulrich bundles with respect to $H$ given by extensions

$0 \rightarrow \mathcal{O}_{X}\left(2 C_{0}+(\beta+g-1) F\right) \otimes \pi^{*} \mathcal{L}_{1} \rightarrow E \rightarrow \mathcal{I}_{Z}\left(2 C_{0}+(2 \beta+g-1-e) F\right) \otimes \pi^{*} \mathcal{L}_{2} \rightarrow 0$ where $\mathcal{L}_{1} \in \mathrm{Pic}^{0}(C)$ is a general line bundle, $\mathcal{L}_{2}=\mathfrak{k} \otimes \mathcal{L}_{1}^{-1}$, and $Z$ a general zerodimensional subscheme of $X$ with $\ell(Z)=\beta-e$.

Proof. The bundles $E$ given by extensions as above fall under the statement of Lemma 3.2: $c_{1}(E) \equiv K_{X}+3 H, c_{2}(E)=4(g-1)+7 \beta-7 e$ and the line bundle $\mathcal{L}_{2}$ is determined by the condition $\operatorname{det}(E)=\mathcal{O}_{X}\left(K_{X}+3 H\right)$. Hence, they are Ulrich if and only if they are initialized.

We have the short exact sequence

$$
0 \rightarrow H^{0}\left(\mathcal{O}_{X}((g-1) F) \otimes \pi^{*} \mathcal{L}_{1}\right) \rightarrow H^{0}(E(-H)) \rightarrow H^{0}\left(\mathcal{I}_{Z}((\beta+g-1-e) F) \otimes \pi^{*} \mathcal{L}_{2}\right) .
$$

For $\mathcal{L}_{1}$ general, $H^{0}\left(\mathcal{O}_{X}((g-1) F) \otimes \pi^{*} \mathcal{L}_{1}\right)=H^{0}\left(\mathcal{O}_{C}((g-1) p) \otimes \mathcal{L}_{1}\right)=0$, as $\mathcal{O}_{C}((g-$ 1) $p) \otimes \mathcal{L}_{1}$ is a general bundle in $\operatorname{Pic}^{g-1}(C)$. For $Z$ general $\pi_{*} \mathcal{I}_{Z}=\mathcal{O}_{C}\left(-\left(p_{1}+\ldots+p_{\beta-e}\right)\right)$ with $p_{i} \in C$ distinct points and, since $\mathcal{L}_{2}=\mathfrak{k} \otimes \mathcal{L}_{1}^{-1}$ is also general, $\pi_{*}\left(\mathcal{I}_{Z}((\beta+g-1-\right.$ e) $F) \otimes \pi^{*} \mathcal{L}_{2}$ ) is a general line bundle of degree $g-1$ and hence has no sections. It follows that $E$ is initialized.

Finally, we consider the remaining cases.

Theorem 3.4. Let $X$ be a geometrically ruled surface over a smooth curve $C$ of genus $g$ and with $e>0$. Let $H_{\alpha}=\alpha C_{0}+\beta F$ with $\alpha \geq 3$ be any very ample divisor on $X$ with

$$
2 \beta>\max \{(\alpha-3)(g-1)+e \alpha,(g-1)+e \alpha\} .
$$

Then, there are special Ulrich bundles with respect to $H_{\alpha}$ given by extensions $0 \rightarrow \mathcal{O}_{X}\left(\alpha C_{0}+(\beta+g-1) F\right) \otimes \pi^{*} \mathcal{L}_{1} \rightarrow E \rightarrow \mathcal{I}_{Z}\left((2 \alpha-2) C_{0}+(2 \beta+g-1-e) F\right) \otimes \pi^{*} \mathcal{L}_{2} \rightarrow 0$ with $\mathcal{L}_{1} \in \operatorname{Pic}^{0}(C)$ a general line bundle, $\mathcal{L}_{2}=\mathfrak{k} \otimes \mathcal{L}_{1}^{-1}$ and $Z$ a general zero-dimensional subscheme of $X$ with $\ell(Z)=(\alpha-1)\left(\beta-\frac{e \alpha}{2}\right)$.

Proof. We proceed in several steps.

Step1. We prove first that there are vector bundles in the extensions like in the statement. To this end, we need to verify that $Z$ verifies the Cayley-Bacharach property with respect to the linear system

$\left.\mid \mathcal{O}_{X}\left(K_{X}+(2 \alpha-2) C_{0}+(2 \beta+g-1-e) F-\alpha C_{0}-(\beta+g-1) F\right) \otimes \pi^{*} \mathcal{L}_{2} \otimes \pi^{*} \mathcal{L}_{1}^{-1}\right) \mid=$ 


$$
\left.=\mid \mathcal{O}_{X}\left(K_{X}+(\alpha-2) C_{0}+(\beta-e) F\right) \otimes \pi^{*}\left(\mathcal{L}_{2} \otimes \mathcal{L}_{1}^{-1}\right)\right) \mid .
$$

Since $\beta>\alpha e>(\alpha-1) e$ and $\alpha \geq 3$, the class $(\alpha-2) C_{0}+(\beta-e) F$ is very ample. Hence, by the Kodaira vanishing

$$
\begin{gathered}
h^{0}\left(\mathcal{O}_{X}\left(K_{X}+(\alpha-2) C_{0}+(\beta-e) F\right) \otimes \pi^{*}\left(\mathcal{L}_{2} \otimes \mathcal{L}_{1}^{-1}\right)\right)= \\
=\chi\left(\mathcal{O}_{X}\left(K_{X}+(\alpha-2) C_{0}+(\beta-e) F\right) \otimes \pi^{*}\left(\mathcal{L}_{2} \otimes \mathcal{L}_{1}^{-1}\right)\right)= \\
=(\alpha-3)\left(g-1+\beta-\frac{e \alpha}{2}\right) .
\end{gathered}
$$

The hypothesis (1), i.e. the condition $2 \beta>(\alpha-3)(g-1)+e \alpha$ implies

$$
h^{0}\left(\mathcal{O}_{X}\left(K_{X}+(\alpha-2) C_{0}+(\beta-e) F\right) \otimes \pi^{*}\left(\mathcal{L}_{2} \otimes \mathcal{L}_{1}^{-1}\right)\right) \leq \ell(Z)-1,
$$

and it follows that for a general $Z$ and any $x \in \operatorname{supp}(Z)$, we have

$$
h^{0}\left(\mathcal{I}_{Z \backslash\{x\}}\left(K_{X}+(\alpha-2) C_{0}+(\beta-e) F\right) \otimes \pi^{*}\left(\mathcal{L}_{2} \otimes \mathcal{L}_{1}^{-1}\right)\right)=0 .
$$

Hence $Z$ trivially satisfies the Cayley-Bacharach property with respect to the linear system

$$
\left.\mid \mathcal{O}_{X}\left(K_{X}+(\alpha-2) C_{0}+(\beta-e) F\right) \otimes \pi^{*}\left(\mathcal{L}_{2} \otimes \mathcal{L}_{1}^{-1}\right)\right) \mid
$$

and therefore, in any extension as in the statement there are rank-two vector bundles. It is clear that the determinant of such a bundle equals $\mathcal{O}_{X}\left(K_{X}+3 H_{\alpha}\right)$. Moreover, a direct computation shows that the second Chern class is

$$
-\frac{\alpha(5 \alpha-3)}{2} e+\beta(5 \alpha-3)+(3 \alpha-2)(g-1)=\frac{1}{2} H_{\alpha} \cdot\left(5 H_{\alpha}+3 K_{X}\right)+2 \chi\left(\mathcal{O}_{X}\right) .
$$

So, it is Ulrich if and only if it is initialized.

Step 2. We prove that the rank two vector bundles we have constructed are initialized. We consider the short exact sequence

$0 \rightarrow H^{0}\left(\mathcal{O}_{X}((g-1) F) \otimes \pi^{*} \mathcal{L}_{1}\right) \rightarrow H^{0}\left(E\left(-H_{\alpha}\right)\right) \rightarrow H^{0}\left(\mathcal{I}_{Z}\left((\alpha-2) C_{0}+(\beta+g-1-e) F\right) \otimes \pi^{*} \mathcal{L}_{2}\right)$.

The genericity of $\mathcal{L}_{1}$ ensures that $H^{0}\left(\mathcal{O}_{X}((g-1) F) \otimes \pi^{*} \mathcal{L}_{1}\right)=0$. The hypothesis $\beta>(g-1)+e \alpha$ implies that the class $\alpha C_{0}+(\beta-g+1) F$ is very ample. Thus, Kodaira vanishing implies

$$
\begin{gathered}
h^{0}\left(\mathcal{O}_{X}\left((\alpha-2) C_{0}+(\beta+g-1-e) F\right) \otimes \pi^{*} \mathcal{L}_{2}\right)=\chi\left(\mathcal{O}_{X}\left((\alpha-2) C_{0}+(\beta+g-1-e) F\right) \otimes \pi^{*} \mathcal{L}_{2}\right)= \\
=-\frac{\alpha(\alpha-1) e}{2}+(\alpha-1) \beta=\ell(Z) .
\end{gathered}
$$

Therefore, for a general $Z$ we have

$$
h^{0}\left(\mathcal{I}_{Z}\left((\alpha-2) C_{0}+(\beta+g-1-e) F\right) \otimes \pi^{*} \mathcal{L}_{2}\right)=0
$$

which finishes the proof.

Remark 3.5. The condition (1) is a very mild one. Besides, if $g=0$ or $g=1$, it is automatically satisfied.

Remark 3.6. A version of Theorem 3.4, with an almost verbatim proof, yields to existence of rank-two Ulrich bundles on Veronese surfaces, which is the content of [ES03] Proposition 5.9. 
Remark 3.7. While the rank two vector bundles of Proposition 3.1 are strictly semistable, the rank two bundles from Proposition 3.3 and Theorem 3.4 are stable with respect to the given polarizations. Indeed, they are semistable and, if they were strictly semistable the the maximal destabilazing sequences would provide us with Ulrich line bundles, CH12, Theorem 2.9 (b)], which cannot exist, Theorem 2.1.

Theorem 3.4 and [Kim16] immediately yield the following

Corollary 3.8. Let $X \stackrel{\sigma}{\rightarrow} \mathbb{P}^{2}$ be the blowup of $\mathbb{P}^{2}$ at $n \geq 1$ general points, denote $E_{1}, \ldots E_{n}$ the exceptional divisors and let $H$ be an ample bundle on $X$ of type $\sigma^{*} \mathcal{O}_{\mathbb{P}^{2}}(k)-$ $\sum E_{i}$. Then there exists a rank-2 Ulrich bundle on $X$ with respect to $H$.

\section{REFERENCES}

[AFO12] M. Aprodu, G. Farkas and A. Ortega, Minimal resolutions, Chow forms and Ulrich bundles on K3 surfaces, preprint, arXiv:1212.6248, to appear in J. Reine Angew. Math.

[Bea00] A. Beauville, Determinantal hypersurfaces, Michigan Math. J. 48 (2000), 39-64

[Bea16] A. Beauville, Ulrich bundles on surfaces with $q=p_{g}=0$, preprint arXiv:1607.00895.

[CH12] M. Casanellas and R. Hartshorne, Stable Ulrich bundles, Int. J. Math. 23 (2012), 1250083

[CKM12] E. Coskun, R. S. Kulkarni, and Y. Mustopa. Pfaffian Quartic Surfaces and Representations of Clifford Algebras, Documenta Math. 17 (2012) 1003-1028.

[CKM13] E. Coskun, R. S. Kulkarni and Y. Mustopa, The geometry of Ulrich bundles on del Pezzo surfaces, J. Algebra 375 (2013), 280301.

[CMR] L. Costa and R. M. Miró-Roig, GL(V)-invariant Ulrich bundles on Grassmannians, Math. Ann. 361 (2015), no. 1-2, 443457.

[CMP] L. Costa, R.M. Miró-Roig and J. Pons-Llopis, The representation type of Segre varieties, Adv. in Math., 230 (2012), 1995-2013.

[ES03] D. Eisenbud and F. -O. Schreyer (with an appendix by J. Weyman), Resultants and Chow forms via exterior syzygies, J. Amer. Math. Soc. 16 (2003), 537-579

[ES11] D. Eisenbud and F. -O. Schreyer, Boij-Söderberg Theory, Combinatorial Aspects of Commutative Algebra and Algebraic Geometry, Abel Symposium 2009, Springer Verlag (2011), 35-48.

[Kim16] Y. Kim. Ulrich bundles on blowups, preprint arXiv:1607.02995

$[\mathrm{KM}] \quad$ J. O. Kleppe and R.M. Miró-Roig, On the normal sheaf of determinantal varieties, J. Reine Angew. Math., Ahead of Print Journal, DOI 10.1515/ crelle-2014-0041.

[Mi] R.M. Miró-Roig, The representation type of rational normal scrolls, Rendiconti del Circolo di Palermo 62 (2013) 153-164.

[MP] R.M. Miró-Roig and J. Pons-Llopis, Representation type of rational ACM surfaces $X \subset \mathbb{P}^{4}$, Algebras and Representation Theory, 16 (2013) 1135-1157.

[MP2] R.M. Miró-Roig and J. Pons-Llopis, $N$-dimensional Fano varieties of wild representation type, Jour. Pure and Applied Alg. 218 (2014), 1867-1884.

[U184] B. Ulrich, Gorenstein Rings and Modules with High Numbers of Generators, Math. Z. 188 (1984), 23-32

Facultatea de Matematică şi Informatică, Universitatea din Bucureşti, Str. Academiei 14, 010014 Bucureşti, ROMAniA \& Institutul De Matematic $\breve{~ " S i m i o n ~ S t o i l o w " ~ A l ~ A c a d e m i e i ~}$ Române, Calea Griviţei 21, Sector 1, 010702 Bucureşti, ROMANiA

E-mail address: marian.aprodu@fmi.unibuc.ro \& marian.aprodu@imar.ro

Facultat de MatemÀtiques i Informàtica, Departament de Matemàtiques i Informàtica, Gran Via de les Corts Catalanes 585, 08007 Barcelona, SPAIN

E-mail address: costa@ub.edu 
FACultat de Matemàtiques i Informàtica, Departament de Matemàtiques i InformàticA, Gran Via de les Corts Catalanes 585, 08007 Barcelona, SPAIN

E-mail address: miro@ub.edu 